\title{
On the number of bouncing ball modes in billiards
}

\author{
A Bäcker†§, R Schubert†\| and P Stifterł $\uparrow$ \\ $\dagger$ Abteilung Theoretische Physik, Universität Ulm, Albert-Einstein-Allee 11, D-89069 Ulm, \\ Germany \\ ‡ Abteilung Quantenphysik, Universität Ulm, Albert-Einstein-Allee 11, D-89069 Ulm, Germany
}

Received 7 May 1997

\begin{abstract}
We study the number of bouncing ball modes $N_{\mathrm{bb}}(E)$ in a class of two-dimensional quantized billiards with two parallel walls. Using an adiabatic approximation we show that asymptotically $N_{\mathrm{bb}}(E) \sim \alpha E^{\delta}$ for $E \rightarrow \infty$, where $\left.\delta \in\right] \frac{1}{2}, 1[$ depends on the shape of the billiard boundary. In particular for the class of two-dimensional Sinai billiards, which are chaotic, one can get arbitrarily close (from below) to $\delta=1$, which corresponds to the leading term in Weyl's law for the mean behaviour of the counting function of eigenstates. This result shows that one can come arbitrarily close to violating quantum ergodicity. We compare the theoretical results with the numerically determined counting function $N_{\mathrm{bb}}(E)$ for the stadium billiard and the cosine billiard and find good agreement.
\end{abstract}

\section{Introduction}

In quantum chaos one is interested in studying properties of quantum-mechanical systems, whose classical limit is chaotic, see for example [1]. In particular, billiard systems have been studied thoroughly because a lot of analytic results are known on the classical ergodic properties. Classically, a two-dimensional Euclidean billiard system is given by the free motion of a point particle inside a domain $\Omega \subset \mathbb{R}^{2}$ with elastic reflections at the boundary $\partial \Omega$. The corresponding quantum-mechanical system is given by the stationary Schrödinger equation $(\hbar=2 m=1)$

$$
\left(\Delta+E_{n}\right) \psi_{n}(x)=0 \quad \forall x \in \Omega \backslash \partial \Omega
$$

with Dirichlet boundary condition $\psi_{n}(\boldsymbol{x})=0$ on the boundary $\partial \Omega$. Much work has been devoted to the investigation of statistical properties of the set of eigenvalues $\left\{E_{n}\right\}$ and eigenfunctions $\left\{\psi_{n}\right\}$ and their dependence on the properties of the classical system.

In this paper we study a special type of eigenfunctions which occur in billiards with two parallel walls (like the stadium billiard), the so-called bouncing ball modes. These are eigenfunctions which are localized in the rectangular part between the parallel walls and have a structure similar to eigenfunctions of a rectangular box. The classical billiard possesses a family of neutral periodic orbits bouncing up and down between the two parallel walls, and the bouncing ball modes are semiclassically concentrated on this family.

Already in [2] bouncing ball-like states were discussed and then were observed in the stadium billiard $[3,4]$. A method of constructing these states approximately was given in

$\S$ E-mail address: baec@physik.uni-ulm.de

|| E-mail address: schub@physik.uni-ulm.de

ๆ E-mail address: stif@physik.uni-ulm.de 
[5]. Based on this work, our principal aim is to count the number of bouncing ball modes up to energy $E$,

$$
N_{\mathrm{bb}}(E):=\left\{n \mid E_{n} \leqslant E \text {, with } \psi_{n} \text { being a bouncing ball state }\right\} .
$$

We will derive the asymptotic behaviour of $N_{\mathrm{bb}}(E)$ for $E \rightarrow \infty$, and compare it with numerical results. For the stadium billiard this question has been addressed in [6] where it is argued that bouncing ball modes exist up to infinite energy, and in [7] the asymptotic behaviour of $N_{\mathrm{bb}}(E)$ has been derived for this system.

For us the main motivation for studying this question comes from quantum ergodicity. Assume that we have a billiard $\Omega$ where the classical flow is ergodic, for example the Sinai billiard [8] or the stadium billiard $[9,10]$. This means that for a particle moving along a generic trajectory, the probability of finding it at time $t$ in some region of phase space is the relative volume of that region. Quantum ergodicity denotes a quantum analogue of this behaviour. One says that a subsequence $\left\{E_{n_{j}}\right\} \subset\left\{E_{n}\right\}$ has density 1 if

$$
\lim _{E \rightarrow \infty} \frac{\#\left\{n_{j} \mid E_{n_{j}} \leqslant E\right\}}{N(E)}=1
$$

where

$$
N(E):=\#\left\{n \mid E_{n} \leqslant E\right\}
$$

is the spectral staircase function, counting the number of energy levels below a given energy $E$. The quantum ergodicity theorem states that if the classical flow is ergodic, then there is a subsequence $\left\{E_{n_{j}}\right\} \subset\left\{E_{n}\right\}$ of density 1 , such that

$$
\lim _{j \rightarrow \infty} \int_{\Omega^{\prime}}\left|\psi_{n_{j}}(\boldsymbol{x})\right|^{2} \mathrm{~d}^{2} x=\frac{\left|\Omega^{\prime}\right|}{|\Omega|}
$$

for every subset $\Omega^{\prime} \subset \Omega$. So for almost all eigenfunctions the probability of finding a particle in a certain region $\Omega^{\prime}$ of the position space $\Omega$ becomes proportional to the volume $\left|\Omega^{\prime}\right|$ if the energy $E$ becomes large. In fact a stronger result is valid, where the left-hand side of (5) is given by an integral of the Wigner function of a state $\psi_{n_{j}}$ over a phase-space region and the right-hand side is given by the relative volume of this region. This concept of quantum ergodicity was introduced for flows on manifolds without boundaries in [11], and was proven in [12-14]. In $[15,16]$ quantum ergodic theorems are proven for a large class of billiard systems, which include for example the stadium billiard.

The condition for the density of the subsequence to be 1 leaves a lot of room for subsequences of eigenstates which are not quantum ergodic in the sense of (5). In general, the behaviour of the counting function of such sequences is an open question; the quantum ergodicity theorem only says that it is of lower order than $N(E)$. It is even conjectured for certain systems that no such exceptional subsequences exist, i.e. relation (5) is valid for the whole sequence $\left\{\psi_{n}\right\}$, a property which is thus called unique quantum ergodicity, see $[13,17]$. The bouncing ball modes clearly form an exceptional subsequence which does not satisfy (5). If the billiard is ergodic and its geometry permits the existence of bouncing ball modes, as it is the case for the stadium and Sinai billiard, then the quantum ergodicity theorem gives an upper bound for $N_{\mathrm{bb}}(E)$,

$$
\lim _{E \rightarrow \infty} \frac{N_{\mathrm{bb}}(E)}{N(E)}=0 .
$$

Any further information on the asymptotic behaviour of $N_{\mathrm{bb}}(E)$ provides information on the question whether one could improve the quantum ergodicity theorem by giving a stronger bound on the number of non-quantum ergodic states. 


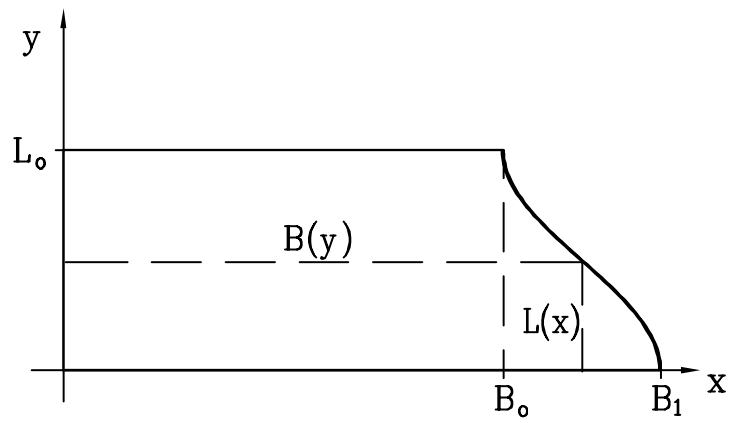

Figure 1. Boundary of the desymmetrized cosine billiard, $B(y)=2+\frac{1}{2}(1+\cos (\pi y))$, and notation used for the adiabatic approximation.

\section{Adiabatic approximation of bouncing ball modes}

The type of billiards we investigate in the following is a class of two-dimensional billiards, where one example is shown in figure 1 . The billiard has the shape of a rectangular box of height $L_{0}$ and width $B_{0}$, at which one side is replaced by a curved boundary, defined by a function $L(x)$ with $L\left(B_{0}\right)=L_{0}, L\left(B_{1}\right)=0$ and $B_{1}>B_{0}$. The function $L(x)$ is assumed to be decreasing for $x>B_{0}$ in a neighbourhood of $x=B_{0}$.

First we have to make the notion of a bouncing ball mode more precise. The idea is that the bouncing ball modes are eigenfunctions which are semiclassically concentrated on the bouncing ball orbits in phase space. Let $\Omega_{B}:=\left\{(x, y) ; x \in\left[0, B_{0}\right], y \in\left[0, L_{0}\right]\right\}$ be the box-like region between the parallel walls in the billiard. We say that a sequence of eigenfunctions $\left\{\psi_{n_{j}}\right\}$ is a sequence of bouncing ball modes, if they satisfy the following two conditions

$$
\begin{aligned}
& \text { (i) } \lim _{j \rightarrow \infty} \operatorname{supp}\left(\psi_{n_{j}}\right)=\Omega_{B} \\
& \text { (ii) } \lim _{j \rightarrow \infty}\left|\widehat{\psi}_{n_{j}}\left(\sqrt{E_{n_{j}}} p_{x}, \sqrt{E_{n_{j}}} p_{y}\right)\right|^{2}=\delta\left(p_{x}\right) \frac{\delta\left(p_{y}-1\right)+\delta\left(p_{y}+1\right)}{2} \text {. }
\end{aligned}
$$

The first condition expresses the localization on the bouncing ball orbits in position space, where $\operatorname{supp}(\psi)$ is the support of $\psi$, i.e. the closure of the set where $\psi$ is not zero. A function which is approximately zero outside $\Omega_{B}$ and satisfies the Schrödinger equation inside $\Omega_{B}$, is close to an eigenfunction for $\Omega_{B}$, so the bouncing ball modes should be close to

$$
\psi_{n_{j}}(x, y)=\sqrt{\frac{2}{B_{0}}} \sin \left(\frac{\pi}{B_{0}}\left(k_{j}+v\right) x\right) \sqrt{\frac{2}{L_{0}}} \sin \left(\frac{\pi l_{j}}{L_{0}} y\right)
$$

with eigenvalues $E_{n_{j}}=\pi^{2}\left(\frac{l_{j}^{2}}{L_{0}^{2}}+\frac{\left(k_{j}+v\right)^{2}}{B_{0}^{2}}\right)$, where $k_{j}, l_{j} \in \mathbb{N} \backslash 0$. Here $v$ is an arbitrary phase shift.

The second condition expresses the localization in momentum space, because the bouncing ball orbits have momentum $p_{x}=0$ and $p_{y}= \pm 1$ on the energy surface $p^{2}=1$. Here $\widehat{\psi}$ denotes the Fourier transform of $\psi$. The arguments have been rescaled by the square root of the energy, because the Fourier transform is concentrated on the energy shell, which is with this scaling a circle of radius 1 .

Consider now a sequence $E_{n_{j}}=\pi^{2}\left(\frac{l_{j}^{2}}{L_{0}^{2}}+\frac{\left(k_{j}+v\right)^{2}}{B_{0}^{2}}\right) \rightarrow \infty$, and the corresponding sequence $\left|\widehat{\psi}_{n_{j}}\left(\sqrt{E_{n_{j}}} p_{x}, \sqrt{E_{n_{j}}} p_{y}\right)\right|^{2}$ with $\psi_{n_{j}}$ given by (8). The computation of $\widehat{\psi}_{n_{j}}$ shows that the 
sequence $\left|\widehat{\psi}_{n_{j}}\right|^{2}$ converges in the limit $n_{j} \rightarrow \infty$, if and only if the sequence $\frac{k_{j} L_{0}}{l_{j} B_{0}}$ converges for $j \rightarrow \infty$, the limit of the latter is then denoted by $\beta$. In this case the sequence $\left|\widehat{\psi}_{n_{j}}\right|^{2}$ tends to a sum of four delta functions concentrated on the unit circle at angles $\pi / 2 \pm \arctan (\beta)$, $3 \pi / 2 \pm \arctan (\beta)$. Therefore condition (ii) of (7) demands that $\beta=0$, or equivalently

$$
\lim _{j \rightarrow \infty} \frac{k_{j}}{l_{j}}=0 .
$$

A consequence of this condition is that the counting function for the bouncing ball modes cannot be of leading order, i.e. equation (6) follows for these modes without assuming ergodicity.

The general picture of a bouncing ball mode at finite energies which emerges from our discussion is a plane wave in two dimensions with frequencies $\left(\frac{\pi k}{B_{0}}, \frac{\pi l}{L_{0}}\right)$, and $l \gg k$. However, for finite energies the eigenfunctions will certainly penetrate the region outside $\Omega_{B}$ because an eigenfunction of the Laplacian in $\Omega$ cannot vanish in an open subset of $\Omega$. Therefore, following [5], we make the adiabatic separation ansatz

$$
\psi_{l, k}(x, y)=\varphi_{l}(y ; x) \chi_{l, k}(x)
$$

with

$$
\varphi_{l}(y ; x)=\sqrt{\frac{2}{L(x)}} \sin \left(\frac{\pi l}{L(x)} y\right)
$$

where $L(x)$ is the width of the billiard at the length $x$ as defined in figure 1 . The function (10) is still close to a separation ansatz, but the weak dependence of $\varphi_{l}$ on $x$ due to $L(x)$ ensures that $\psi_{l, k}$ satisfies Dirichlet boundary conditions on $\partial \Omega$.

The function $\varphi_{l}$ can be viewed as quantization of the fast motion in $y$-direction. The slow motion in $x$-direction is then according to [5] quantized by demanding that $\chi_{l, k}$ satisfies the Schrödinger equation

$$
-\chi_{l, k}^{\prime \prime}(x)+l^{2} V(x) \chi_{l, k}(x)=e_{l, k} \chi_{l, k}(x)
$$

with the potential

$$
V(x)=\frac{\pi^{2}}{L^{2}(x)}-\frac{\pi^{2}}{L_{0}^{2}}
$$

From the eigenvalues $e_{l, k}$ of (12) one obtains the eigenvalues $E_{l, k}=\frac{l^{2} \pi^{2}}{L_{0}^{2}}+e_{l, k}$ of the Schrödinger equation (1).

The ansatz (10) satisfies the Schrödinger equation (1) exactly inside the rectangular region $\Omega_{B}$. Since the potential $l^{2} V(x)$ becomes very steep for large $l$, the two directions will be effectively decoupled outside $\Omega_{B}$ for $l \gg k$. So (10) satisfies the Schrödinger equation (1) if $l \gg k$.

The main point now is to estimate how large $k$ may be for a given $l$. The principal idea is that the de Broglie wavelength in the $x$-direction should be large compared with the effective length how far $\psi_{l, k}$ penetrates into the region outside $\Omega_{B}$, because then the wavefunction does not feel the deviation of $l^{2} V(x)$ from an infinitely high wall. Therefore we first have to derive some asymptotic properties of the solutions to (12).

The potential $V(x)$ is zero for $x \leqslant B_{0}$ and tends to infinity for $x \rightarrow B_{1}$, see figure 2 . So for $x<B_{0}$ the $\chi_{l, k}$ are plane waves with frequency $\sqrt{e_{l, k}}$.

Equation (12) is of WKB type with WKB parameter $l$. Define $x_{l, k}^{0}$ by

$$
l^{2} V\left(B_{0}+x_{l, k}^{0}\right)=e_{l, k} .
$$




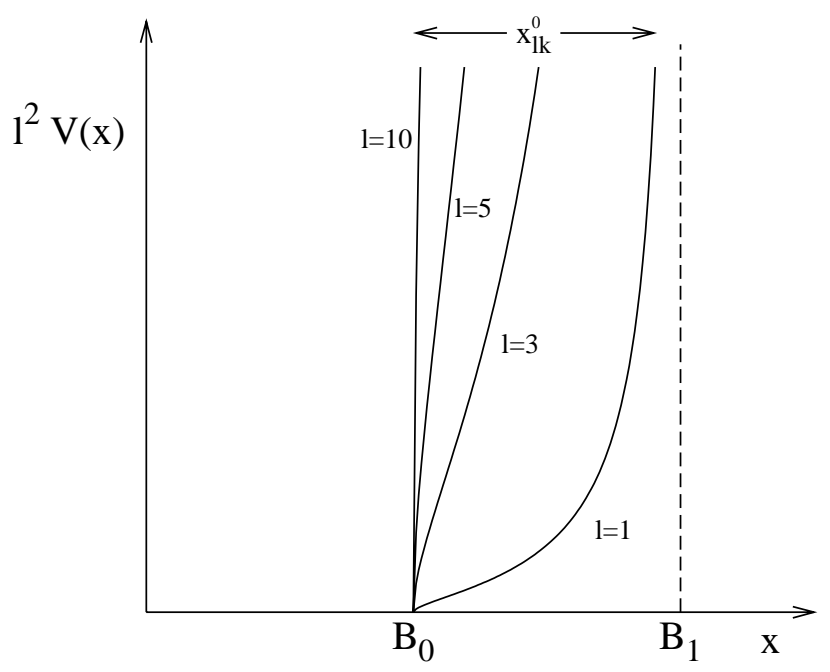

Figure 2. Potential $l^{2} V(x)$ in the $x$-direction for the adiabatic approximation.

Standard WKB theory (see, e.g. [18]) predicts that the solution $\chi_{l, k}$ to equation (12) is exponentially small for $x>B_{0}+x_{l, k}^{0}$ in the limit $l \rightarrow \infty$, so one only has to care about the interval $\left[B_{0}, B_{0}+x_{l, k}^{0}\right]$. Since we are looking for solutions which asymptotically live in $\Omega_{B}$ we assume that $x_{l, k}^{0} \rightarrow 0$ for $E \rightarrow \infty$. Then we only need to know the potential $V$ in a small neighbourhood of $B_{0}$. For $x \searrow 0$ one can write $L(x)$ as

$$
L\left(B_{0}+x\right) \sim L_{0}-C x^{\gamma}
$$

with characteristic exponent $\gamma>0$, and $C>0$. For example, for the stadium billiard one has $\gamma=2$ and for the cosine and the Sinai billiard (with circular hole) $\gamma=\frac{1}{2}$. The potential $V(x)$ is thus for $x \searrow B_{0}$ given by

$$
V\left(B_{0}+x\right) \sim c x^{\gamma}
$$

with $c=2 \pi^{2} C / L_{0}^{3}$. In particular, for $x_{l, k}^{0}$ defined in (14) we obtain

$$
x_{l, k}^{0} \sim\left(\frac{e_{l, k}}{c l^{2}}\right)^{\frac{1}{\gamma}} \quad \text { for } l \rightarrow \infty .
$$

For the eigenvalues $e_{l, k}$ one has the WKB condition

$$
\begin{aligned}
k-\frac{1}{2} & =\frac{1}{\pi} \int_{0}^{B_{0}+x_{l, k}^{0}} \sqrt{e_{l, k}-l^{2} V(x)} \mathrm{d} x \\
& =\frac{B_{0}}{\pi} \sqrt{e_{l, k}}+\frac{1}{\pi} \int_{0}^{x_{l, k}^{0}} \sqrt{e_{l, k}-l^{2} V\left(B_{0}+x\right)} \mathrm{d} x .
\end{aligned}
$$

Inserting expression (16), and substituting $x=y\left(\frac{e_{l, k}}{c l^{2}}\right)^{-\frac{1}{\gamma}}=\frac{y}{x_{l, k}^{0}}$, leads to

$$
\sqrt{e_{l, k}}=\frac{\pi}{B_{0}}\left(k-\frac{1}{2}\right)-\frac{\pi}{B_{0}} \sqrt{e_{l, k}} x_{l, k}^{0} \int_{0}^{1} \sqrt{1-x^{\gamma}} \mathrm{d} x .
$$

Now we return to the main question, to consider for which $l, k$ the ansatz (10) satisfies the Schrödinger equation. By construction the ansatz has the correct boundary conditions, and satisfies the Schrödinger equation inside $\Omega_{B}$. By $x_{l, k}^{0}$ the depth how far the functions 
(10) penetrate into the region outside $B_{0}$ is characterized, and the de Broglie wavelength of $\psi_{l, k}$ in the $x$-direction is $\lambda_{l, k}=2 \pi / \sqrt{e_{l, k}}$. If we demand that the ratio between $x_{l, k}^{0}$ and the de Broglie wavelength tends to zero for large energies, then as mentioned above, $\psi_{l, k}$ will satisfy the Schrödinger equation (1). With $\lambda_{l, k}=2 \pi / \sqrt{e_{l, k}}$ this condition leads to

$$
\lim _{E_{j} \rightarrow \infty} \sqrt{e_{l_{j}, k_{j}}} x_{l_{j}, k_{j}}^{0}=0 .
$$

Using this condition formula (19) gives for the $e_{l, k}$ the asymptotic expression

$$
e_{l, k}=\frac{\pi^{2}}{B_{0}^{2}}\left(k-\frac{1}{2}\right)^{2} \text {. }
$$

Then (20), together with (17) and (21), leads to

$$
\lim _{l, k \rightarrow \infty} \frac{k^{\frac{2}{\gamma}+1}}{l^{\frac{2}{\gamma}}}=0 .
$$

This is the crucial condition which tells us how fast $k$ may grow compared with $l$ and which in turn gives us the number of bouncing ball modes which exist up to a given energy $E$. So

$$
k_{\max } \propto l^{\frac{2}{\gamma+2}}
$$

is the borderline below which our ansatz satisfies asymptotically the Schrödinger equation with the energy

$$
E_{l, k}=\frac{\pi^{2}}{L_{0}^{2}} l^{2}+e_{l, k}=\frac{\pi^{2}}{L_{0}^{2}} l^{2}+\frac{\pi^{2}}{B_{0}^{2}}\left(k-\frac{1}{2}\right)^{2} .
$$

The number of such states up to energy $E$ asymptotically equals the area of the intersection between an ellipse with axes $\sqrt{E} L_{0} / 2, \sqrt{E} B_{0} / 2$, and the domain between the curve $l^{\frac{2}{2+\gamma}}$ and the $x$-axis. This area asymptotically equals

$$
\int_{0}^{\sqrt{E} L_{0} / 2} l^{\frac{2}{2+\gamma}} \mathrm{d} l=\text { const } E^{\delta}
$$

with exponent (recall that $\gamma$ is defined by equation (15))

$$
\delta=\frac{1}{2}+\frac{1}{2+\gamma} .
$$

So we have found an upper bound for the number of bouncing ball modes in the sense that

$$
N_{\mathrm{bb}}(E)=\mathrm{o}\left(E^{\delta}\right)
$$

but

$$
\lim _{E \rightarrow \infty} \frac{E^{\delta-\epsilon}}{N_{\mathrm{bb}}(E)}=0 \quad \text { for all } \epsilon>0 .
$$

This means $E^{\delta}$ is the smallest power which is an upper bound for $N_{\mathrm{bb}}(E)$.

Note that the exponent (26) takes its values in $] \frac{1}{2}, 1\left[\right.$, so $N_{\mathrm{bb}}(E)$ varies between the behaviour of the counting function $N(E)$ for a one- and a two-dimensional system. A small exponent $\gamma$ corresponds to a sharp edge at $B_{0}$, where one would intuitively expect more bouncing ball modes as for large $\gamma$, which is confirmed by (26). The two limiting cases $\delta \rightarrow 1$ and $\delta \rightarrow \frac{1}{2}$ correspond to the cases of a right angle and of infinite continuation of the parallel walls respectively. In both cases we would expect the result from direct computations. 
In the case of the stadium billiard the exponent $\delta=\frac{3}{4}$ was previously obtained in [7] by means of an approximation of the semiclassical transfer operator in the bouncing ball limit, which leads to a similar cut-off condition such as (23). We will return to this point in section 3.

The result (27), (28) for $N_{\mathrm{bb}}(E)$ follows from the cut-off condition (20). That this condition should be satisfied is a heuristic argument, one could also imagine that it might suffice that the ratio $x_{l, k}^{0} / \lambda_{l, k}$ tends to some small constant. Then one would obtain $N_{\mathrm{bb}}(E) \sim \alpha E^{\delta}$. But we do not see any reasonable choice for such a constant not being equal to zero.

Concerning equation (21) we should remark that one can obtain better asymptotic expressions by including higher-order terms. For our purpose this is not necessary.

We finish this section by discussing some generalizations of our construction.

The result can immediately be extended to more general geometries of the billiard. Suppose the billiard has a rectangular part, i.e. a region where the opposite sides are parallel straight lines. Similarly one defines the function $L(x)$ as the distance between the opposite sides of the billiard measured orthogonal to the parallel walls. Let the behaviour of $L(x)$ at the endpoints $B_{1}, B_{2}$ of the rectangular region be given by $L\left(B_{1}-x\right)=L_{0}-c_{1} x^{\gamma_{1}}$, and $L\left(B_{2}+x\right)=L_{0}-c_{2} x^{\gamma_{2}}$, with $c_{1}, c_{2}>0$, for small $x$. Then one gets for the exponent $\delta=\frac{1}{2}+1 /\left(2+\gamma_{\max }\right)$, with $\gamma_{\max }=\max \left(\gamma_{1}, \gamma_{2}\right)$.

Our derivation can also be performed for other boundary conditions; the result does not change because the boundary conditions are incorporated in $\varphi_{l}$ which then has to be adapted. For example for Neumann boundary conditions one takes

$$
\varphi_{l}(y ; x)=\sqrt{\frac{2}{L(x)}} \cos \left(\frac{\pi l}{L(x)} y\right)
$$

and equation (12) for $\chi_{l, k}$ remains unchanged. Therefore we get the same asymptotic behaviour for the number of bouncing ball modes in billiards with Neumann boundary conditions as for billiards with Dirichlet boundary conditions. Similarly one can treat the case of mixed boundary conditions, which are discussed in detail in [19].

A particularly nice example is given by the class of Sinai billiards [8] which are given by the free motion in a square with a convex hole (in quantum chaos the term Sinai billiard is usually used for the special case with a circular hole). The class of Sinai billiards is ergodic and one can find for every $\gamma>0$ a Sinai billiard such that $L(x)$ has the characteristic exponent $\gamma$. As an example, consider the case that the boundary curve of the convex hole is given by

$$
\left|L_{0}-L(x)\right|^{\mu}+\left(B_{0}+1-x\right)^{2}=1 \quad \mu \in \mathbb{R}^{+} .
$$

This curve is a circle for $\mu=2$ and tends to a square in the limit $\mu \rightarrow \infty$. In this case we have $\gamma=1 / \mu$, and $\delta=1-\frac{1}{2(2 \mu+1)}$ tends to 1 for $\mu \rightarrow \infty$. Therefore one can find for every $\delta<1$, arbitrarily close to 1 , an ergodic billiard with this $\delta$ giving the asymptotic behaviour of the number of non-quantum ergodic bouncing ball modes. So our analysis shows that the quantum ergodicity theorem cannot be improved without further assumptions on the system.

\section{Numerical results}

As model systems to study the increase of the number of bouncing ball modes numerically, we have chosen the desymmetrized cosine billiard with $B_{0}=2, B_{1}=L_{0}=1$, $B(y)=2+\frac{1}{2}(1+\cos (\pi y))$, see figure 1 , and the desymmetrized stadium billiard with 

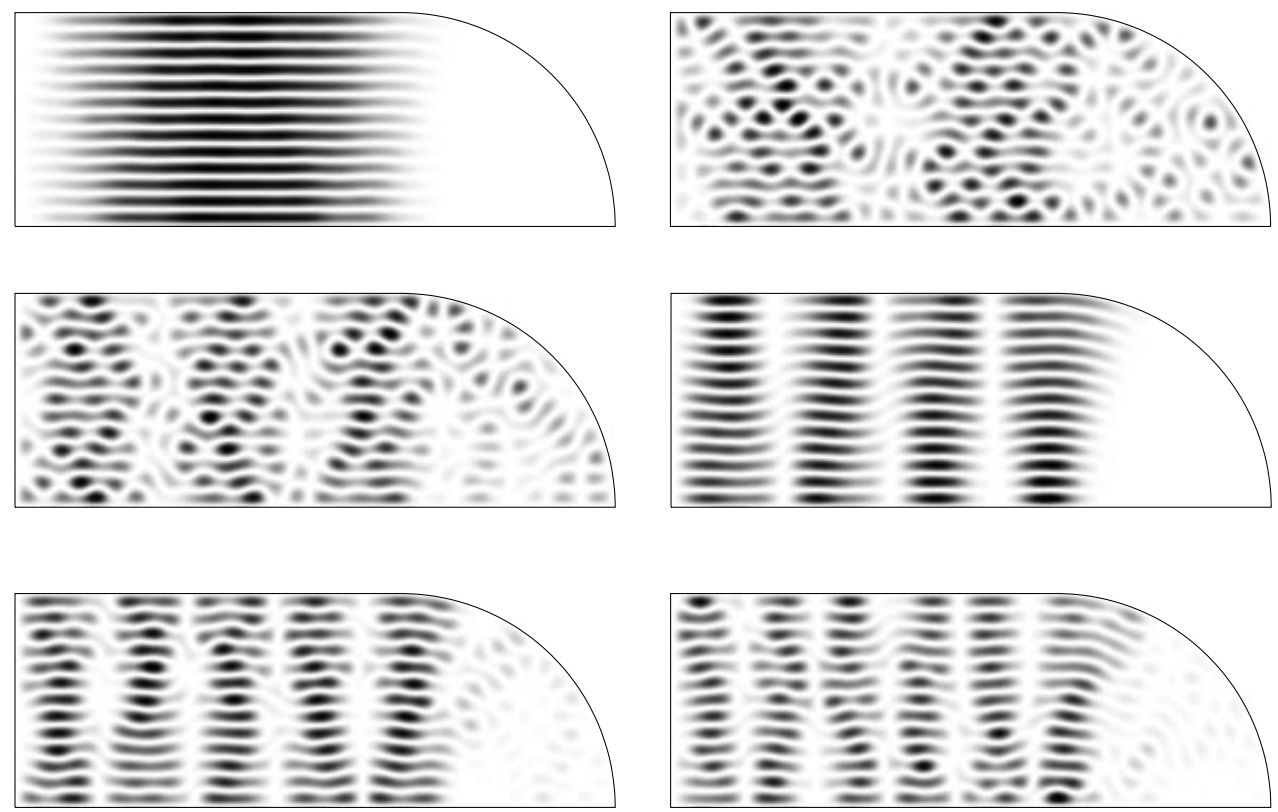

Figure 3. Density plot of $\left|\psi_{n}(x, y)\right|^{2}$ for a series of bouncing ball modes in the stadium billiard, with $n=320(13,1), n=321(13,2), n=325(13,3), n=329(13,4), n=333(13,5)$ and $n=339(13,6)$. The numbers in brackets denote the adiabatic quantum numbers $(l, k)$ being the number of modes in the $x$ - and $y$-direction, respectively. Notice that the second and third bouncing ball mode do not have such a clear structure as the others.

$L_{0}=1, B_{0}=1.8, B_{1}=2.8$, see figure 3 . For the stadium billiard it is proven that it is ergodic, mixing and a $K$-system $[9,10]$. For the cosine billiard numerical studies do not reveal any stability islands; therefore these do not have any influence on the energy range under consideration [20].

A crucial step in the determination of $N_{\mathrm{bb}}(E)$ is the selection of the bouncing ball modes. This selection process was done by visual inspection of the sets of eigenfunctions, according to the description given at the beginning of section 2 . The eigenmodes are found to be organized in series with fixed number $l$ of maxima in $y$-direction, while the number $k$ of maxima in the $x$-direction increases from 1 up to a certain maximum $k_{\max }(l)$. However, we found that there is no sharp border in $k$ within a series; in particular the association was not unambiguous for states with a high number of horizontal nodal lines. Therefore we used a conservative approach, i.e. in doubtful cases we did not count a state as a bouncing ball state.

In order to study the asymptotic behaviour of the bouncing ball modes, we first selected the bouncing ball modes from the first 2000 (odd-odd symmetry) eigenfunctions of the stadium billiard and from the first 1620 (odd-odd symmetry) eigenfunctions of the cosine billiard. In both cases the eigenfunctions were obtained by the boundary integral method [21,22] using a singular-value decomposition [23].

As an example in figures 3 and 4 we show density plots of $\left|\psi_{n}\right|^{2}$ of a complete series of bouncing ball modes for both systems. Notice that some states strongly resemble states of a rectangular billiard, whereas there are also states in between, which do not have such a clear structure. Moreover, we observed gaps (i.e. missing $k$ values) in several series of bouncing ball modes with fixed number of vertical nodal lines, which was also remarked in [7]. 

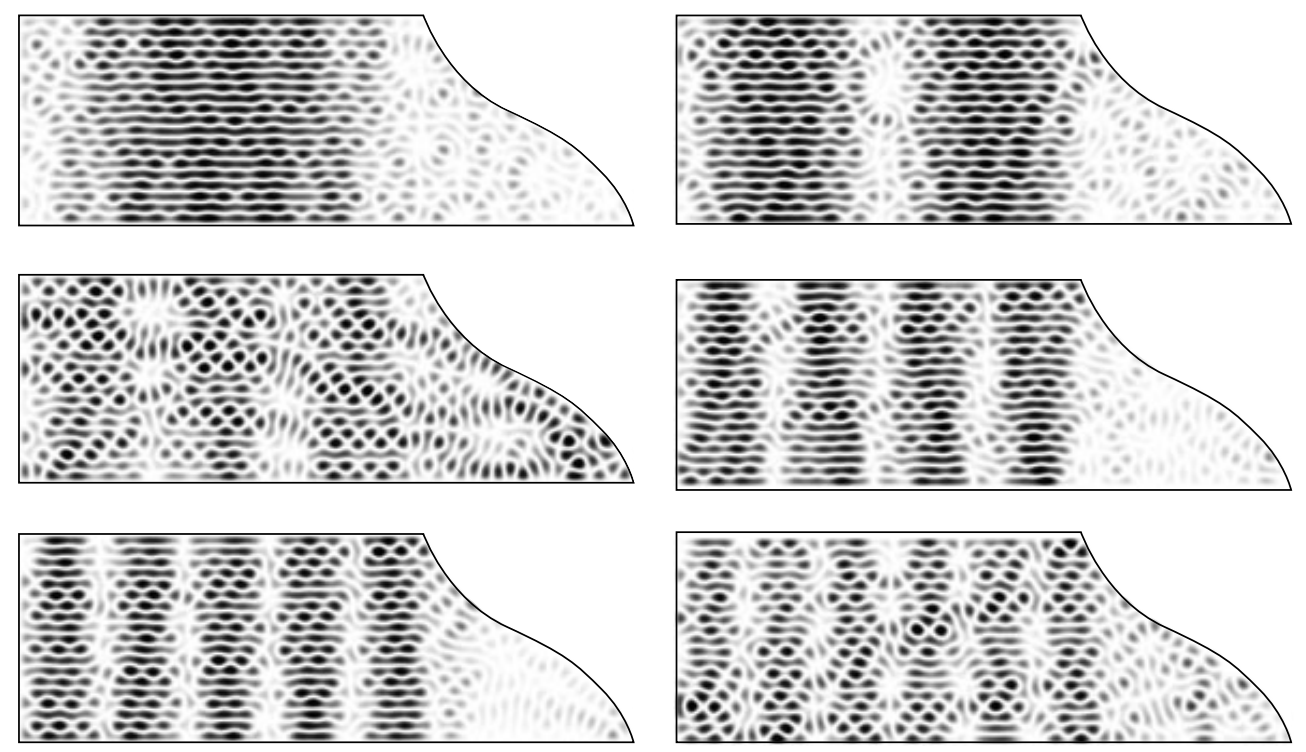

Figure 4. Density plot of $\left|\psi_{n}(x, y)\right|^{2}$ for a series of bouncing ball modes for the cosine billiard, with $n=673(19,1), n=675(19,2), n=678(19,3), n=682(19,4), n=687(19,5)$ and $n=691(19,6)$.

These observations can be explained by taking the neighbouring eigenfunctions into account. In the case of the missing bouncing ball modes we found that these can be recovered as a superposition of eigenfunctions whose eigenvalues are close to the one of the expected bouncing ball mode. The involved eigenfunctions themselves do not resemble the bouncing ball mode structure, see figures $5(d)-5(f)$. If the eigenvalue of a bouncing ball state is nearly degenerated, i.e. the nearest neighbouring eigenvalue is much closer than the mean level spacing, we find that the bouncing ball mode structure is less clear. Instead the bouncing ball mode structure is distributed among these two states, see figures $5(a)-5(c)$ illustrating this for the $(13,2)$ bouncing ball mode in the stadium billiard (see also figure 3 ). By varying the parameter $B_{0}$ in the stadium billiard we found that this phenomenon is due to avoided crossings of the corresponding energy levels. Since the mean level spacing is independent of the energy for two-dimensional billiards, we expect the fraction of the missing bouncing ball modes to be constant. Therefore their contribution only affects the prefactor $\alpha$ in equation (31) below, but not the exponent $\delta$.

Among the first 2000 odd-odd eigenstates of the stadium we identified 224 bouncing ball modes, from which we obtain $N_{\mathrm{bb}}(E)$ as shown in figure 6 . In addition, a fit to

$$
N_{\mathrm{bb}}(E)=\alpha E^{\delta}
$$

is shown with $\alpha=0.20$ and $\delta=0.76$. Similarly we obtain $\delta=0.72$ for the stadium billiard with even-even symmetry using the first 2000 eigenstates. As already mentioned, in the case of the stadium billiard the exponent $\delta=\frac{3}{4}$ for $N_{\mathrm{bb}}(E)$ has also been derived in [7]. Additionally the derivation in [7] provides asymptotically the factor $\alpha=\frac{a}{\pi^{3 / 2}}=0.323 \ldots$, which, however, does not agree with our numerical result. One might think that this is due to the missing bouncing ball modes, but their inclusion in the counting function increases $\alpha$ only slightly. We see two possible reasons for this disagreement of the prefactor: first, the result in [7] is based on an estimate of the volume of the region in phase space around the 
(a)

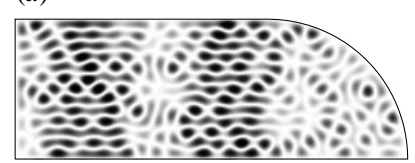

(d)

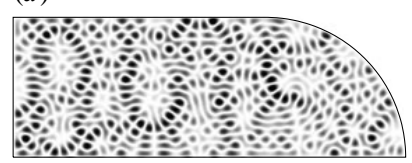

(b)

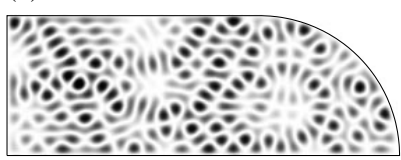

(e)

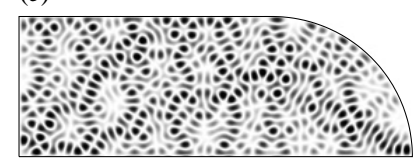

(c)
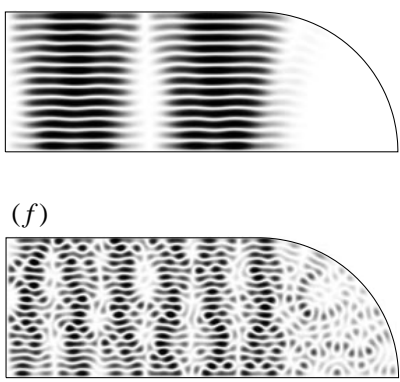

Figure 5. Examples showing that by superposition of two states the structure of not so clear bouncing ball modes can be drastically improved $(c)$, or missing bouncing ball modes can be recovered $(f)$. Density plot $(c)$ shows the superposition $\psi^{+}$of $(a), n=321$ (see also figure 3), with the next eigenfunction $(b), n=322$. In this case $\psi^{+}=\cos (0.2 \pi) \psi_{321}+\sin (0.2 \pi) \psi_{322}$. The second superposition f) is given by $\psi^{+}=\cos (0.2 \pi) \psi_{718}+\sin (0.2 \pi) \psi_{719}$, with $\psi_{718}$ and $\psi_{719}$ shown in $(e)$ and $(d)$, respectively. Notice that $\psi_{718}$ and $\psi_{719}$ do not resemble any bouncing ball mode structure. In both cases the corresponding eigenvalues have a separation which is much smaller than the mean level spacing.

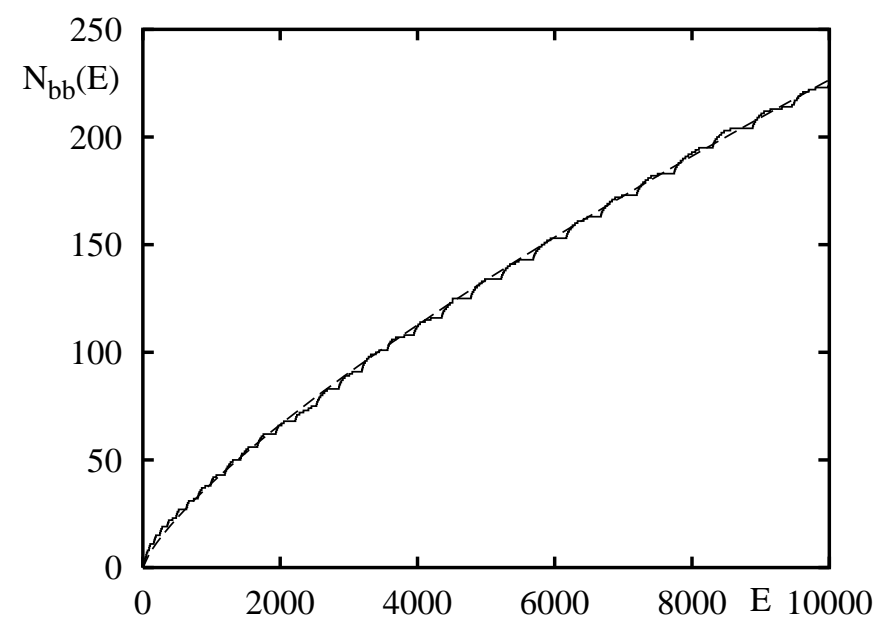

Figure 6. $N_{\mathrm{bb}}(E)$ for the stadium billiard together with the fit $\alpha E^{\delta}$, where $\alpha=0.20$ and $\delta=0.76$.

bouncing ball orbits which can be considered regular at energy $E$. We observe a rather large number of eigenfunctions which are scarred by periodic orbits lying in a neighbourhood of the bouncing ball orbits in phase space. If we include these eigenfunctions, we obtain a prefactor $\alpha=0.27$ (and exponent $\delta=0.753$ ), which is still smaller than the result of Tanner. Secondly, next-to-leading-order contributions could influence the result of the fit.

From the first 1620 eigenstates of the cosine billiard 137 bouncing ball modes have been selected. The result for $N_{\mathrm{bb}}(E)$ is shown in figure 7. Also shown is a fit to equation (31) with an additional constant $\beta$ starting from energy $E=800$, giving $\alpha=0.04, \delta=0.87$ and $\beta=12.4$. The reason for performing the fit not from $E=0$ but from $E=800$ is clearly visible in figure 7. For smaller energies $N_{\mathrm{bb}}(E)$ has a different slope than for larger energies. This presumably is due to next-to-leading-order contributions to $N_{\mathrm{bb}}(E)$, which 


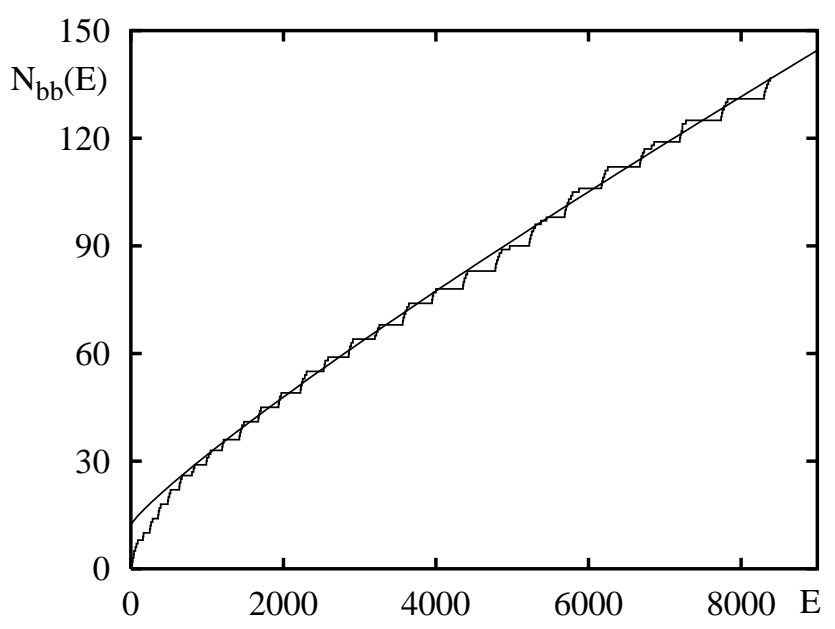

Figure 7. $N_{\mathrm{bb}}(E)$ for the cosine billiard together with the fit $\alpha x^{\delta}+\beta$ where $\alpha=0.04, \delta=0.87$ and $\beta=12.4$.

may dominate the low-energy range.

The oscillatory contribution of the bouncing ball orbits to the spectral staircase function (first observed in [24]) has been derived in [25]; to avoid confusion we should remark that the function $\hat{N}_{b}(k)$ in [25] is not the counting function for the bouncing ball modes.

For both systems we find good agreement of the numerically determined exponent $\delta$ with the corresponding results $\delta=\frac{3}{4}$ for the stadium and $\delta=\frac{9}{10}$ for the cosine billiard. This agreement is quite remarkable for two reasons: first, equation (31) is only an asymptotic result for $N_{\mathrm{bb}}(E)$ and thus there could be corrections dominating the low-energy domain; secondly, as already mentioned, there is the problem of identifying bouncing ball modes with a large number of horizontal nodal lines, because visually there is no clear border between bouncing ball modes and others.

We should remark that the total number of observed bouncing ball modes in the considered energy range is smaller for the cosine billiard than for the stadium billiard, despite the fact that the exponent $\delta$ is larger for the cosine billiard; the reason for this lies in the smaller prefactor $\alpha$ in case of the cosine billiard. However, that the system with larger exponent has a smaller prefactor is probably accidental, we see no reason why this should be the case in general. It would be interesting to test this for other billiards with $\delta=\frac{9}{10}$, for example a Sinai billiard with a circular or elliptical hole.

\section{Summary}

In this paper we have studied the number of bouncing ball modes $N_{\mathrm{bb}}(E)$ in Euclidean billiards. We have presented a derivation using the adiabatic separation ansatz of [5] of the asymptotic behaviour for $N_{\mathrm{bb}}(E)$. For two-dimensional systems our result is

$$
N_{\mathrm{bb}}(E) \sim \alpha E^{\delta} \quad \text { for } E \rightarrow \infty
$$

where $\delta$ varies between $\frac{1}{2}$ and 1 , depending on the geometry of the billiard. For example for the stadium billiard one gets $\delta=\frac{3}{4}$, and for the Sinai billiard with a circular hole and the cosine billiard $\delta=\frac{9}{10}$. In fact any billiard whose boundary is locally of circular or elliptic shape at the connection point $B_{0}$ with the rectangular part has $\delta=\frac{9}{10}$. We 
have checked the exponent numerically for the stadium and the cosine billiard, and the agreement with the theoretical results is even at low energies very good. Furthermore, we found that missing bouncing ball modes and the occurrence of states with a not so clear bouncing ball mode structure are due to avoided level crossings. Varying the length $B_{0}$ of the billiard the bouncing ball mode structure is passed in an avoided level crossing from one eigenfunction to the other. We illustrated that by superpositions of neighbouring states the missing bouncing ball modes can be recovered.

Our derivation applied to the class of two-dimensional Sinai billiards with a convex hole gives that one can find for every $\frac{1}{2}<\delta<1$ an ergodic billiard for which (32) holds. This shows that the bound on the number of non-quantum ergodic eigenfunctions in ergodic billiards, as given by the quantum ergodicity theorem, cannot be relaxed in general.

\section{Acknowledgments}

We would like to thank Professor Dr F Steiner, Dr R Aurich, Dr J Bolte, Dr M Sieber and Dr G Tanner for useful discussions and comments. We also would like to thank the referees for useful comments. AB acknowledges support by the Deutsche Forschungsgemeinschaft under contract no DFG-Ste 241/7-2.

\section{References}

[1] Gutzwiller M C 1990 Chaos in Classical and Quantum Mechanics (New York: Springer)

[2] Keller J B and Rubinow S I 1960 Asymptotic solution of eigenvalue problems Ann. Phys., NY 9 24-75

Keller J B and Rubinow S I 1960 Asymptotic solution of eigenvalue problems Ann. Phys., NY $10303-5$ (erratum)

[3] McDonald S W and Kaufmann A N 1988 Wave chaos in the stadium: statistical properties of short-wave solutions of the Helmholtz equation Phys. Rev. A 37 3067-86

[4] Heller E J 1984 Bound-state eigenfunctions of classically chaotic Hamiltonian systems: scars of periodic orbits Phys. Rev. Lett. 53 1515-18

[5] Bai Y Y, Hose G, Stefański K and Taylor H S 1985 Born-Oppenheimer adiabatic mechanism for regularity of states in the quantum stadium billiard Phys. Rev. A 31 2821-6

[6] O'Connor P W and Heller E J 1988 Quantum localization for a strongly classically chaotic system Phys. Rev. Lett. $612288-91$

[7] Tanner G 1997 How chaotic is the stadium billiard? A semiclassical analysis J. Phys. A: Math. Gen. 30 2863-88

[8] Sinai Ya G 1970 Dynamical systems with elastic reflections Russ. Math. Surv. 25 137-89

[9] Bunimovich L A 1974 On ergodic properties of certain billiards Funct. Anal. Appl. 8 254-5

[10] Bunimovich L A 1979 On the ergodic properties of nowhere dispersing billiards Commun. Math. Phys. 65 295-312

[11] Shnirelman A I 1974 Ergodic properties of eigenfunctions Usp. Math. Nauk 29 181-2 (in Russian)

[12] Shnirelman A I 1993 On the asymptotic properties of eigenfunctions in the regions of chaotic motion (addendum to V F Lazutkin) KAM Theory and Semiclassical Approximations to Eigenfunctions (Berlin: Springer)

[13] Colin de Verdière Y 1985 Ergodicité et fonctions propres du laplacien Commun. Math. Phys. 102 111-34

[14] Zelditch S 1987 Uniform distribution of eigenfunctions on compact hyperbolic surfaces Duke. Math. J. 55 919-41

[15] Gérard P and Leichtnam E 1993 Ergodic properties of eigenfunctions for the Dirichlet problem Duke Math. J. 71 559-607

[16] Zelditch S and Zworski M 1996 Ergodicity of eigenfunctions for ergodic billiards Commun. Math. Phys. 175 $673-82$

[17] Sarnak P 1995 Arithmetic quantum chaos Isr. Math. Conf. Proc. 8 183-236

[18] Landau L D and Lifshitz E M 1977 Quantum Mechanics: Non-relativistic Theory 3rd edn (Oxford: Pergamon)

[19] Sieber M, Primack H, Smilansky U, Ussishkin I and Schanz H 1995 Semiclassical quantization of billiards with mixed boundary conditions J. Phys. A: Math. Gen. 28 5041-78 
[20] Stifter P 1996 Quantenbillards und Quantenchaos $P h D$ thesis Abteilung für Quantenphysik, Universität Ulm

[21] Riddel J R Jr 1979 Boundary-distribution solution of the Helmholtz equation for a region with corners $J$. Comp. Phys. 31 21-41

[22] Berry M V and Wilkinson M 1984 Diabolical points in the spectra of triangles Proc. Roy. Soc. London 392 15-43

[23] Aurich R and Steiner F 1993 Statistical properties of highly excited quantum eigenstates of a strongly chaotic system Physica 64D 185-214

[24] Gräf H-D, Harney H L, Lengeler H, Lewenkopf C H, Rangacharyulu C, Richter A, Schardt P and Weidenmüller H A 1992 Distribution of eigenmodes in a superconducting stadium billiard with chaotic dynamics Phys. Rev. Lett. 69 1296-9

[25] Sieber M, Smilansky U, Creagh S C and Littlejohn R G 1993 Non-generic spectral statistics in the quantized stadium billiard J. Phys. A: Math. Gen. 26 6217-30 